\title{
Doubly heavy baryons and quark-diquark symmetry in quenched and partially quenched chiral perturbation theory
}

\author{
Thomas Mehen ${ }^{1,2, *}$ and Brian C. Tiburzi ${ }^{1, \dagger}$ \\ ${ }^{1}$ Department of Physics, Duke University, Durham, North Carolina 27708, USA \\ ${ }^{2}$ Jefferson Laboratory, 12000 Jefferson Ave., Newport News, Virginia 23606, USA \\ (Received 21 July 2006; published 21 September 2006)
}

\begin{abstract}
We extend the chiral Lagrangian with heavy quark-diquark symmetry to quenched and partially quenched theories. These theories are used to derive formulas for the chiral extrapolation of masses and hyperfine splittings of double heavy baryons in lattice QCD simulations. A quark-diquark symmetry prediction for the hyperfine splittings of heavy mesons and doubly heavy baryons is rather insensitive to chiral corrections in both quenched and partially quenched QCD. Extrapolation formulas for the doubly heavy baryon electromagnetic transition moments are also determined for the partially quenched theory.

\section{INTRODUCTION}

Two heavy antiquarks in a doubly heavy antibaryon feel an attractive force when they are in the $\mathbf{3}$ of color $S U(3)$ and therefore are expected to form a compact diquark whose size, $r_{Q Q} \sim\left(m_{Q} v\right)^{-1}$, is much smaller than $\Lambda_{\mathrm{QCD}}^{-1}$. Here $m_{Q}$ is the heavy quark mass and $v$ is the typical velocity of the antiquarks in the diquark. To the light degrees of freedom in the doubly heavy antibaryon, this diquark is indistinguishable from a heavy quark in the $m_{Q} \rightarrow \infty$ limit. Therefore, the properties of ground state doubly heavy baryons should be related to properties of singly heavy mesons. Savage and Wise [1] first observed that quark-diquark symmetry is a consequence of the heavy quark limit and used heavy quark effective theory (HQET) [2] to derive a relation between hyperfine splittings of doubly heavy baryons and singly heavy mesons. ${ }^{1}$ The correct framework for analyzing the properties of hadrons with two or more heavy quarks is nonrelativistic QCD (NRQCD) [5-7] and recently the heavy quark-diquark symmetry prediction was rederived in both the potential NRQCD [8] and velocity NRQCD [9] formalisms. An extension of heavy hadron chiral perturbation theory $(\mathrm{HH} \chi \mathrm{PT})$ [10-12] that incorporates heavy quark-diquark symmetry was developed in Ref. [13] and used to derive a novel quark-diquark symmetry prediction for the electromagnetic decays of the spin-3/2 members of the ground state doubly heavy baryon doublets, as well as study $O\left(1 / m_{Q}\right)$ and chiral corrections to the electromagnetic decays and doubly heavy baryon masses. Some quarkdiquark symmetry predictions for the exotic heavy tetraquarks as well as a critical discussion of the applicability of heavy quark-diquark symmetry to charm hadrons appeared in Ref. [14].

\footnotetext{
*Electronic address: mehen@phy.duke.edu

$\dagger$ Electronic address: bctiburz@phy.duke.edu

${ }^{1}$ The quark model has also been used to derive the relation $[3,4]$.
}

Much of the recent theoretical work has been stimulated by the SELEX collaboration reports of candidates for doubly charm baryons [15-17]. While the masses and hyperfine splittings of the observed states are consistent with theoretical calculations in the quark model [4] as well as quenched lattice QCD, other aspects of the data are poorly understood at the present time. For example, isospin splittings, weak decay rates, and production yields are in disagreement with theoretical expectations. Some excited states which would be expected to decay electromagnetically are instead observed via their weak decay. For a review of the experimental situation and discussion of theoretical interpretations of the SELEX results, see Refs. [13,14,18]. Recently, the BABAR [19] and BELLE [20] experiments searched for doubly charmed baryons in $e^{+} e^{-}$collisions but failed to observe the SELEX states. More experimental data and improved theoretical understanding of doubly charm baryons are necessary to clarify the situation.

Lattice gauge theory studies of the properties of doubly heavy baryons with dynamical quarks would be quite useful. First principles calculation of the spectroscopy of doubly heavy baryons could be directly compared with experiment to test our understanding of QCD and aid in the interpretation of candidate states. It would also be of interest to have first principle calculations of electromagnetic and weak decay matrix elements. Finally, the lattice could help determine the suitability of quark-diquark symmetry for charm and bottom hadrons. In these cases, it is possible that symmetry breaking corrections could be large enough to spoil symmetry predictions, but no systematic study of symmetry breaking corrections in NRQCD is yet available. A theoretical testing ground in which the heavy quark mass can be dialed at will could determine whether this symmetry will turn out to be useful for charm and bottom. Spectroscopic studies of doubly charm and bottom baryons in quenched lattice QCD appeared in Refs. [3,21,22]. The heavy quark-diquark symmetry prediction for the hyperfine splitting holds at the $20 \%$ level, 
which is reasonable given the sources of error. In QCD, corrections are expected to be $O\left(v^{2}\right)$ and/or $O\left(\Lambda_{\mathrm{QCD}} / m_{Q}\right)$. In existing lattice calculations there are also uncontrolled errors due to quenching, which, as we will see below, could be significant. Hopefully, the near future will bring studies of doubly heavy baryons in simulations with dynamical quarks.

As a step towards such studies, it is clearly useful to develop extensions of the heavy quark-diquark symmetric version of $\mathrm{HH} \chi \mathrm{PT}$ [13] that include lattice artifacts such as quenching and partial quenching. These theories provide the formulas needed for chiral extrapolations in lattice calculations. We will focus on chiral corrections to the doubly heavy baryon masses, especially hyperfine splittings and the quark-diquark symmetry prediction. We also apply the formalism to the electromagnetic decays of the spin-3/2 members of the ground state doubly heavy baryon doublets.

The organization of our paper is as follows. In Sec. II, we review the basics of quenched chiral perturbation theory $(\mathrm{Q} \chi \mathrm{PT})$ and partially quenched chiral perturbation theory $(\mathrm{PQ} \chi \mathrm{PT})$ in the pseudoscalar meson sector. We then write down versions of $\mathrm{PQ} \chi \mathrm{PT}$ and $\mathrm{Q} \chi \mathrm{PT}$ that incorporate heavy quark-diquark symmetry. In Sec. III, we calculate the one-loop chiral corrections to the masses of doubly heavy baryons in PQ $\chi \mathrm{PT}$ and $\mathrm{Q} \chi \mathrm{PT}$. These expressions can be used to perform the chiral extrapolation of lattice data on the spectrum of doubly heavy baryons provided one is in the range of applicability of the chiral effective theory. In Sec. IV, we calculate to one loop the dipole and quadrupole transition moments in $\mathrm{PQ} \chi \mathrm{PT}$. For these transitions, the lattice can be used to test the predictions for the electromagnetic decay widths of the spin-3/2 members of the ground state doubly heavy baryon doublets [13]. Finally, we conclude with a brief summary in Sec. V. Some useful formulas are collected in the appendix.

\section{PQ $\chi$ PT AND $Q \chi P T$ FOR DOUBLY HEAVY BARYONS}

The formalism for constructing effective theories for quenched and partially quenched QCD is now well established. We use the technique of graded Lie algebras that was formulated in Refs. [23-30]. Equivalently one could use the replica method [31]. We begin by briefly reviewing the pseudoscalar meson sector of $\mathrm{Q} \chi \mathrm{PT}$ and $\mathrm{PQ} \chi \mathrm{PT}$, and then write down the quenched and partially quenched chiral Lagrangians incorporating heavy quark-diquark symmetry. These theories are an extension of $\mathrm{Q} \chi \mathrm{PT}$ and $\mathrm{PQ} \chi \mathrm{PT}$ for singly heavy mesons [32].

\section{A. Pseudoscalar mesons}

In the pseudoscalar meson sector, we can treat $\mathrm{PQ} \chi \mathrm{PT}$ and $\mathrm{Q} \chi \mathrm{PT}$ as different limits of the same theory. This theory is described by the Lagrangian

$$
\begin{aligned}
\mathcal{L}= & \frac{f^{2}}{8} \operatorname{str}\left(\partial^{\mu} \Sigma^{\dagger} \partial_{\mu} \Sigma\right)+\frac{\lambda}{4} \operatorname{str}\left(m_{q} \Sigma^{\dagger}+m_{q}^{\dagger} \Sigma\right) \\
& +\alpha_{\Phi} \partial^{\mu} \Phi_{0} \partial_{\mu} \Phi_{0}-\mu_{0}^{2} \Phi_{0}^{2},
\end{aligned}
$$

where the field $\Sigma$ is defined by

$$
\Sigma=\exp \left(\frac{2 i \Phi}{f}\right)=\xi^{2},
$$

and the meson fields appear in the $U(6 \mid 3)$ matrix,

$$
\Phi=\left(\begin{array}{cc}
M & \chi^{\dagger} \\
\chi & \tilde{M}
\end{array}\right)
$$

The quantities $\alpha_{\Phi}$ and $\mu_{0}$ are nonvanishing in the chiral limit. The $M$ and $\tilde{M}$ matrices contain bosonic mesons (with quantum numbers of $q \bar{q}$ pairs and $\tilde{q} \overline{\tilde{q}}$ pairs, respectively, where $q$ is a valence or sea quark and $\tilde{q}$ is a ghost quark), while the $\chi$ and $\chi^{\dagger}$ matrices contain fermionic mesons (with quantum numbers of $\tilde{q} \bar{q}$ pairs and $q \overline{\tilde{q}}$ pairs, respectively). The upper $3 \times 3$ block of the matrix $M$ contains the familiar pions, kaons, and eta, while the remaining components consist of mesons formed from one or two sea quarks. The operation $\operatorname{str}()$ in Eq. (1) is a supertrace over flavor indices, i.e., $\operatorname{str}(A)=\sum_{a} \epsilon_{a} A_{a a}$, where $\epsilon_{a}=$ $(-)^{1+\eta_{a}}$, and $\eta_{a}$ are the grading factors of the Lie algebra.

The quark mass matrix appearing above is given by

$$
m_{q}=\operatorname{diag}\left(m_{u}, m_{d}, m_{s}, m_{j}, m_{l}, m_{r}, m_{u}, m_{d}, m_{s}\right) \text {. }
$$

We will work in the isospin limit of both the valence and sea sectors, where $m_{d}=m_{u}$ and $m_{l}=m_{j}$. Expanding the Lagrangian in Eq. (1) to lowest order in the fields, one finds that mesons with quark content $q \bar{q}^{\prime}$ are canonically normalized and their masses are given by

$$
m_{q q^{\prime}}^{2}=\frac{\lambda}{f^{2}}\left(m_{q}+m_{q^{\prime}}\right)
$$

The propagators of the off-diagonal mesons have the usual Klein-Gordon form.

On the flavor diagonal, the situation is more complicated and differs dramatically between the quenched and partially quenched theories. The flavor singlet field that appears above is defined to be $\Phi_{0}=\operatorname{str}(\Phi) / \sqrt{6}$. Using this, the leading-order $\eta_{a} \eta_{b}$ propagator is [29]

$$
\begin{aligned}
\mathcal{G}_{a b}\left(q^{2}\right)= & \frac{i \epsilon_{a} \delta_{a b}}{q^{2}-m_{a a}^{2}+i \epsilon} \\
& -\frac{i}{3} \frac{\left(q^{2}-m_{j j}^{2}\right)\left(q^{2}-m_{r r}^{2}\right)\left(\alpha_{\Phi} q^{2}-\mu_{0}^{2}\right)}{\left(q^{2}-m_{a a}^{2}+i \epsilon\right)\left(q^{2}-m_{b b}^{2}+i \epsilon\right) D\left(q^{2}\right)} .
\end{aligned}
$$

The function $D\left(q^{2}\right)$ is defined as

$$
D\left(q^{2}\right)=\left(q^{2}-m_{j j}^{2}\right)\left(q^{2}-m_{r r}^{2}\right)+\left(\alpha_{\Phi}^{2} q^{2}-\mu_{0}^{2}\right)\left(q^{2}-m_{X}^{2}\right),
$$

and the mass $m_{X}$ is given by $m_{X}^{2}=\frac{1}{3}\left(m_{j j}^{2}+2 m_{r r}^{2}\right)$. 
Because the fermionic determinant in quenched QCD is set to a constant, the sea quark masses are effectively infinite. The mesons containing sea quarks decouple from the theory, leaving a $U(3 \mid 3)$ Goldstone manifold. In this limit, the flavor neutral propagator in Eq. (6) becomes

$$
\begin{aligned}
\mathcal{G}_{a b}^{Q}\left(q^{2}\right)= & \frac{i \epsilon_{a} \delta_{a b}}{q^{2}-m_{a a}^{2}+i \epsilon} \\
& -\frac{i}{3} \frac{\alpha_{\Phi} q^{2}-\mu_{0}^{2}}{\left(q^{2}-m_{a a}^{2}+i \epsilon\right)\left(q^{2}-m_{b b}^{2}+i \epsilon\right)},
\end{aligned}
$$

where we have appended a superscript $Q$ to denote that this propagator is for the quenched theory. Notice the flavor singlet meson does not decouple as the theory still depends on the parameters $\alpha_{\Phi}$ and $\mu_{0}$. Numerical data suggest that $\alpha_{\Phi}$ is small (for example, a recent study finds $\alpha_{\Phi}=$ $0.03 \pm 0.03$ [33]), and we neglect it below; $\mu_{0}$ will be treated in the standard quenched power counting [34].

In the partially quenched theory, sea quark contributions are retained and partially quenched QCD (PQQCD) has a strong axial anomaly. Because of this, the mass of the singlet field $\mu_{0}$ can be taken to be on the order of the chiral symmetry breaking scale, and the $\Phi_{0}$ integrated out of the theory. The Goldstone manifold is reduced to $S U(6 \mid 3)$ but the resulting flavor neutral two-point function, however, still deviates from the familiar form in $\chi \mathrm{PT}$. In PQQCD this propagator is given by the $\mu_{0} \rightarrow \infty$ limit of Eq. (6), namely

$$
\mathcal{G}_{a b}^{\mathrm{PQ}}\left(q^{2}\right)=\frac{i \epsilon_{a} \delta_{a b}}{q^{2}-m_{a a}^{2}+i \epsilon}-\frac{i}{3} \frac{\left(q^{2}-m_{j j}^{2}\right)\left(q^{2}-m_{r r}^{2}\right)}{\left(q^{2}-m_{a a}^{2}+i \epsilon\right)\left(q^{2}-m_{b b}^{2}+i \epsilon\right)\left(q^{2}-m_{X}^{2}+i \epsilon\right)}
$$

The PQ $\chi$ PT flavor neutral propagator can be conveniently rewritten as

$$
\mathcal{G}_{a b}^{\mathrm{PQ}}=\epsilon_{a} \delta_{a b} P_{a}+\mathcal{P}_{a b}\left(P_{a}, P_{b}, P_{X}\right),
$$

where

$$
\begin{aligned}
P_{a}= & \frac{i}{q^{2}-m_{a a}^{2}+i \epsilon}, \\
P_{b}= & \frac{i}{q^{2}-m_{b b}^{2}+i \epsilon}, \\
P_{X}= & \frac{i}{q^{2}-m_{X}^{2}+i \epsilon}, \\
\mathcal{P}_{a b}(A, B, C)= & -\frac{1}{3}\left[\frac{\left(m_{a a}^{2}-m_{j j}^{2}\right)\left(m_{a a}^{2}-m_{r r}^{2}\right)}{\left(m_{a a}^{2}-m_{b b}^{2}\right)\left(m_{a a}^{2}-m_{X}^{2}\right)} A\right. \\
& +\frac{\left(m_{b b}^{2}-m_{j j}^{2}\right)\left(m_{b b}^{2}-m_{r r}^{2}\right)}{\left(m_{b b}^{2}-m_{a a}^{2}\right)\left(m_{b b}^{2}-m_{X}^{2}\right)} B \\
& \left.+\frac{\left(m_{X}^{2}-m_{j j}^{2}\right)\left(m_{X}^{2}-m_{r r}^{2}\right)}{\left(m_{X}^{2}-m_{a a}^{2}\right)\left(m_{X}^{2}-m_{b b}^{2}\right)} C\right] .
\end{aligned}
$$

\section{B. Doubly heavy baryons}

As discussed earlier, in the heavy quark limit the properties of singly heavy mesons and doubly heavy baryons are related by a $U(5)$ quark-diquark symmetry [1]. Recently a chiral Lagrangian incorporating this $U(5)$ symmetry has been derived [13]. It is written in terms of a superfield $\mathcal{H}$ given by

$$
\mathcal{H}_{a, \mu \beta}=H_{a, \alpha \beta}+T_{a, i \beta},
$$

where $a$ is an antifundamental flavor index, $\alpha$ and $\beta$ are Pauli spinor indices, while $i$ is a vector spin index. The superspin index $\mu=1-5$ and is related to $\alpha$ and $i$ via

$$
\mu=\alpha\left(\delta_{\mu}^{1}+\delta_{\mu}^{2}\right)+(i+2)\left(\delta_{\mu}^{3}+\delta_{\mu}^{4}+\delta_{\mu}^{5}\right) .
$$

The field $H_{a, \alpha \beta}$ is the heavy meson field of $\mathrm{HH} \chi \mathrm{PT}[10-$ $12]$ in the rest frame of the heavy meson:

$$
H_{a, \alpha \beta}=\boldsymbol{P}_{a}^{*} \cdot \boldsymbol{\sigma}_{\alpha \beta}+P_{a} \delta_{\alpha \beta} .
$$

$H_{a}$ contains the vector $\left(\boldsymbol{P}^{*}\right)$ and pseudoscalar $(P)$ field components. The field $T_{a, i \beta}$ is the doubly heavy baryon field,

$$
T_{a, i \beta}=\sqrt{2}\left(\Xi_{a, i \beta}^{*}+\frac{1}{\sqrt{3}} \Xi_{a, \gamma} \sigma_{\gamma \beta}^{i}\right),
$$

that contains the spin-1/2 ( $\Xi)$ and spin-3/2 ( $\left.\Xi^{*}\right)$ field components. The spin-3/2 field satisfies a nonrelativistic Rarita-Schwinger constraint, $\Xi_{a, i \beta}^{*} \sigma_{\beta \gamma}^{i}=0$.

In terms of the superfield $\mathcal{H}$, the Lagrangian for the quenched $(Q)$ theory is

$$
\begin{aligned}
\mathcal{L}^{Q}= & \left(\mathcal{H}^{\dagger}\left(\mathcal{H} i \stackrel{\leftarrow}{D_{0}}\right)\right)-g^{Q}\left(\mathcal{H}^{\dagger} \mathcal{H} \boldsymbol{A} \cdot \boldsymbol{\sigma}\right) \\
& -g^{\prime Q}\left(\mathcal{H}^{\dagger} \mathcal{H} \boldsymbol{\sigma}\right) \cdot \operatorname{str}(\boldsymbol{A})+\frac{\Delta_{H}^{Q}}{4}\left(\mathcal{H}^{\dagger} \boldsymbol{\Sigma} \cdot \mathcal{H} \boldsymbol{\sigma}\right) \\
& +\sigma^{Q}\left(\mathcal{H}^{\dagger} \mathcal{H} \mathcal{M}\right)+\sigma^{\prime Q}\left(\mathcal{H}^{\dagger} \mathcal{H}\right) \operatorname{str}(\mathcal{M}),
\end{aligned}
$$

while that for the partially quenched theory (PQ) is

$$
\begin{aligned}
\mathcal{L}^{\mathrm{PQ}}= & \left(\mathcal{H}^{\dagger}\left(\mathcal{H} i \stackrel{\leftarrow}{D_{0}}\right)\right)-g^{\mathrm{PQ}}\left(\mathcal{H}^{\dagger} \mathcal{H} \boldsymbol{A} \cdot \boldsymbol{\sigma}\right) \\
& +\frac{\Delta_{H}^{\mathrm{PQ}}}{4}\left(\mathcal{H}^{\dagger} \boldsymbol{\Sigma} \cdot \mathcal{H} \boldsymbol{\sigma}\right)+\sigma^{\mathrm{PQ}}\left(\mathcal{H}^{\dagger} \mathcal{H} \mathcal{M}\right) \\
& +\sigma^{\mathrm{PQ}}\left(\mathcal{H}^{\dagger} \mathcal{H}\right) \operatorname{str}(\mathcal{M}) .
\end{aligned}
$$

Both Lagrangians include the leading quark-diquark symmetry breaking term. The bracket () notation denotes the contraction of flavor, spinor and superspin indices of field 
bilinears. For a generic superspin matrix $X$, flavor matrix $Y$, and spin matrix $Z$, () denotes

$$
\left(\mathcal{H}^{\dagger} X \mathcal{H} Y Z\right)=\sum_{\mu, \nu} \sum_{\alpha, \beta} \sum_{a, b} \epsilon_{a} \mathcal{H}_{a, \mu \alpha}^{\dagger} X_{\mu \nu} \mathcal{H}_{b, \nu \beta} Y_{b a} Z_{\beta \alpha}
$$

In Eqs. (15) and (16), $\boldsymbol{A}$ is the spatial part of the axialvector pion field, $A_{\mu}=\frac{i}{2}\left(\xi \partial_{\mu} \xi^{\dagger}-\xi^{\dagger} \partial_{\mu} \xi\right)$, and $\mathcal{M}$ is the mass operator defined by $\mathcal{M}=\frac{1}{2}\left(\xi m_{q} \xi+\xi^{\dagger} m_{q} \xi^{\dagger}\right)$. The $U(5)$ superspin matrix $\boldsymbol{\Sigma}$ is given by

$$
\boldsymbol{\Sigma}_{\mu \nu}=\left(\begin{array}{cc}
\boldsymbol{\sigma}_{\alpha \beta} & 0 \\
0 & \mathcal{T}_{j k}
\end{array}\right),
$$

where $\left(\mathcal{T}^{i}\right)_{j k}=-i \epsilon_{i j k}$.

Notice that there are separate Lagrangians for quenched and partially quenched QCD. The low-energy constants of the two theories are different, e.g. $g^{\mathrm{PQ}} \neq g^{Q}$. Notice also that in $\mathrm{Q} \chi \mathrm{PT}$ the flavor singlet field cannot be integrated out. Consequently $\operatorname{str}(\boldsymbol{A}) \neq 0$ and the axial hairpin coupling $g^{\prime Q}$ remains. Additionally in $\mathrm{Q} \chi \mathrm{PT}, \operatorname{str}(\mathcal{M})=0$ to leading order, and so we can effectively set $\sigma^{\prime Q}=0$ for our calculations. There is no sector of $\mathrm{Q} \chi \mathrm{PT}$ that contains $\chi \mathrm{PT}$, so in general quenched observables are unrelated to QCD observables. The sea sector of PQQCD is QCD, and hence PQ $\chi$ PT contains the low-energy constants of $\chi \mathrm{PT}$. Restricting the flavor indices to the sea sector in Eq. (16), we recover the chiral Lagrangian of Ref. [13], so $g^{\mathrm{PQ}}, \Delta_{H}^{\mathrm{PQ}}$, $\sigma^{\mathrm{PQ}}$ and $\sigma^{/ \mathrm{PQ}}$ are identical to the low-energy constants $g$, $\Delta_{H}, \sigma$, and $\sigma^{\prime}$ of $\chi \mathrm{PT}$, respectively. At this order there are no additional operators in the partially quenched Lagrangian. Carrying out the superspin sums in Eqs. (15) and (16), we produce separate terms for heavy mesons and doubly heavy baryons but with the same low-energy constants. The heavy meson sector of the $\mathrm{PQ} \chi \mathrm{PT}$ and $\mathrm{Q} \chi \mathrm{PT}$ Lagrangians above is that of Ref. [32].

\section{MASSES}

In this section, we use the quenched and partially quenched chiral Lagrangians that incorporate quarkdiquark symmetry to calculate chiral corrections to the masses of doubly heavy baryons. The results can be used for the chiral extrapolation of lattice data. PQ $\chi \mathrm{PT}$ is considered in subsection III A and $\mathrm{Q} \chi \mathrm{PT}$ is discussed in subsection III B. It is important to keep in mind that while $\mathrm{PQ} \chi \mathrm{PT}$ actually contains $\chi \mathrm{PT}$, there is no such connection between $\mathrm{Q} \chi \mathrm{PT}$ and $\chi \mathrm{PT}$.

Near the chiral limit, the masses of doubly heavy baryons generically have the form

$$
\begin{aligned}
& M_{\Xi}=M_{0}-\frac{1}{2} \Delta_{H}+M_{\Xi}^{(1)}+M_{\Xi}^{(3 / 2)}+\ldots \\
& M_{\Xi^{*}}=M_{0}+\frac{1}{4} \Delta_{H}+M_{\Xi^{*}}^{(1)}+M_{\Xi^{*}}^{(3 / 2)}+\ldots,
\end{aligned}
$$

where $M_{0}$ is the mass of the doubly heavy baryons in the chiral and heavy quark limit and $\Delta_{H}$ is the hyperfine mass splitting of the heavy mesons in the chiral limit. ${ }^{2}$ The hyperfine splitting of the $\Xi^{*}$ and $\Xi$ baryons is $\frac{3}{4} \Delta_{H}$ in the chiral limit due to quark-diquark symmetry. The term $M^{(1)}$ is proportional to $m_{q}$ and comes from tree-level counterterms. The term $M^{(3 / 2)}$ comes from the one-loop diagrams and depends on $m$, the Goldstone boson mass, and $\delta$, the mass splitting between the external and virtual doubly heavy baryons. Goldstone boson masses are $\propto$ $\sqrt{m_{q}}$, while $\delta$ receives contributions from $S U(3)$ breaking counterterms, which are $\propto m_{q}$, and/or hyperfine splittings, which scale as $1 / m_{Q}$. In our power counting we take $\delta \sim$ $m \sim Q$ in which case $M^{(3 / 2)} \propto Q^{3}$. In the chiral and heavy quark limits, $\delta$ can be neglected relative to $m$ and then $M^{(3 / 2)} \propto m_{q}^{3 / 2}$. The ellipsis denotes higher-order terms in the $Q$ expansion.

\section{A. PQ $\chi$ PT}

At linear order in the quark mass, the contributions to the masses of doubly heavy baryons at tree level come from the mass operators in Eq. (16):

$$
M_{\Xi_{a}^{*}}^{(1)}=M_{\Xi_{a}}^{(1)}=-\sigma\left(m_{q}\right)_{a}-\sigma^{\prime} \operatorname{str}\left(m_{q}\right),
$$

where $\operatorname{str}\left(m_{q}\right)=2 m_{j}+m_{r}$ in the isospin limit, and the subscript $a$ denotes the light-quark antifundamental index of the doubly heavy baryon. Combining these results with the mass splitting in the chiral limit, we then define the baryon mass splittings

$$
\Delta_{b a}=M_{\Xi_{b}}-M_{\Xi_{a}}=M_{\Xi_{b}^{*}}-M_{\Xi_{a}^{*}}=\sigma\left[\left(m_{q}\right)_{a}-\left(m_{q}\right)_{b}\right]
$$

$$
\Delta_{b a}^{*}=M_{\Xi_{b}^{*}}-M_{\Xi_{a}}=\frac{3}{4} \Delta_{H}+\sigma\left[\left(m_{q}\right)_{a}-\left(m_{q}\right)_{b}\right] .
$$

At one-loop order, the doubly heavy baryons receive mass contributions in $\mathrm{PQ} \chi \mathrm{PT}$ from the sunset diagrams shown in Fig. 1. The vertices in these diagrams are generated from the pion-baryon interaction terms in Eq. (16), while the hairpin interaction corresponds to the disconnected part of the flavor neutral propagator in Eq. (8). Calculating these $\mathrm{PQ} \chi \mathrm{PT}$ loop diagrams, we find

\footnotetext{
${ }^{2}$ Strictly speaking $\Delta_{H}$ is not same the parameter appearing in the Lagrangian above, Eq. (16), which in this footnote we denote by $\Delta_{H}^{0}$. This parameter, which transforms as a chiral singlet, is corrected by a string of operators that differ only by scaling with powers of $\Delta_{H}^{0} / \Lambda_{\chi}$. Thus we subsume this dependence as $\Delta_{H}=$ $\Delta_{H}\left(\Delta_{H}^{0}\right)$ including also contributions to $\Delta_{H}$ arising from loop graphs. In this way we obtain $\Delta_{H}$ as the chiral limit value. The same is true of all other low-energy constants in Eq. (16). We similarly drop their polynomial dependence on $\Delta_{H}^{0} / \Lambda_{\chi}$, and absorb contributions from loop graphs to work with the values of the low-energy constants in the chiral limit. For more details see, e.g. Ref [35].
} 

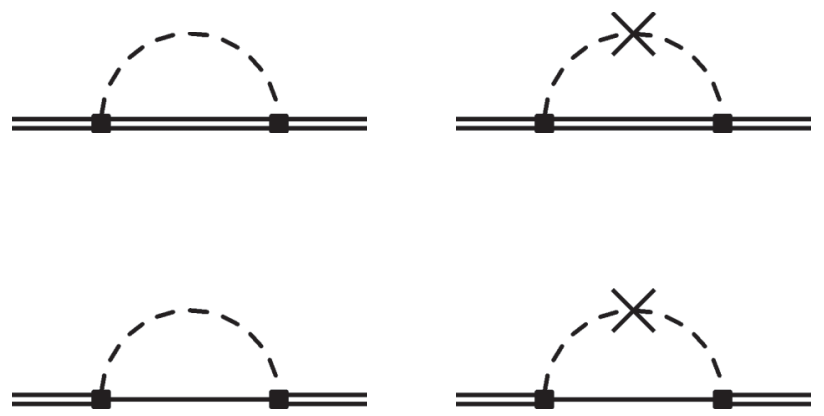

FIG. 1. One-loop graphs which give contributions to the masses of the doubly heavy baryons in PQ $\chi \mathrm{PT}$. The single and double lines correspond to $\Xi$ baryons or $\Xi^{*}$ baryons, respectively, while the dashed lines correspond to mesons. The filled squares denote the nonsinglet axial coupling given in Eq. (16), while crosses denote the hairpin interaction.

$$
\begin{aligned}
M_{\Xi_{a}^{*}}^{(3 / 2)}= & \frac{g^{2}}{(4 \pi f)^{2}} \sum_{b=j, l, r}\left[\frac{5}{9} K\left(\Delta_{b a}, m_{b a}, \mu\right)\right. \\
& \left.+\frac{4}{9} K\left(-\Delta_{a b}^{*}, m_{b a}, \mu\right)\right] \\
& +\frac{g^{2}}{(4 \pi f)^{2}}\left[\frac{5}{9} \mathcal{K}\left(0, m_{a a}, m_{a a}, \mu\right)\right. \\
& \left.+\frac{4}{9} \mathcal{K}\left(-\Delta_{a a}^{*}, m_{a a}, m_{a a}, \mu\right)\right],
\end{aligned}
$$

for the spin-3/2 doubly heavy baryons, $\Xi_{a}^{*}$, and

$$
\begin{aligned}
M_{\Xi_{a}}^{(3 / 2)}= & \frac{g^{2}}{(4 \pi f)^{2}} \sum_{b=j, l, r}\left[\frac{1}{9} K\left(\Delta_{b a}, m_{b a}, \mu\right)\right. \\
& \left.+\frac{8}{9} K\left(\Delta_{b a}^{*}, m_{b a}, \mu\right)\right] \\
& +\frac{g^{2}}{(4 \pi f)^{2}}\left[\frac{1}{9} \mathcal{K}\left(0, m_{a a}, m_{a a}, \mu\right)\right. \\
& \left.+\frac{8}{9} \mathcal{K}\left(\Delta_{a a}^{*}, m_{a a}, m_{a a}, \mu\right)\right],
\end{aligned}
$$

for the spin-1/2 doubly heavy baryons, $\Xi_{a}$. The nonanalytic function $K(\delta, m, \mu)$ is

$$
\begin{aligned}
K(\delta, m, \mu)= & 2\left(\delta^{2}-m^{2}\right)\left[m R\left(\frac{\delta}{m}\right)-\delta \log \left(\frac{m^{2}}{\mu^{2}}\right)\right] \\
& +\delta m^{2} \log \left(\frac{m^{2}}{\mu^{2}}\right)
\end{aligned}
$$

where $R(x)$ is defined by

$$
R(x)=\sqrt{x^{2}-1} \log \left(\frac{x-\sqrt{x^{2}-1+i \epsilon}}{x+\sqrt{x^{2}-1+i \epsilon}}\right),
$$

while the function $\mathcal{K}\left(\delta, m_{a a}, m_{b b}, \mu\right)$ which arises from hairpins is given by

$$
\begin{aligned}
\mathcal{K}\left(\delta, m_{a a}, m_{b b}, \mu\right)= & \mathcal{P}_{a b}\left[K\left(\delta, m_{a a}, \mu\right), K\left(\delta, m_{b b}, \mu\right),\right. \\
& \left.K\left(\delta, m_{X}, \mu\right)\right],
\end{aligned}
$$

with $\mathcal{P}_{a b}$ appearing in Eq. (10), and a limit is understood when $b=a$.

To study the chiral nonanalytic behavior of doubly heavy baryon mass splittings in PQ $\chi \mathrm{PT}$, we need values for the low-energy constants. We choose $g=0.6$ [36], $\Delta_{H}=140 \mathrm{MeV}$, and $f=130 \mathrm{MeV}$. The $S U(3)$ splitting of the ground state $D$ mesons is $\approx 100 \mathrm{MeV}$. Assuming the same $S U(3)$ splitting for the doubly charm baryons we obtain $\sigma / \lambda=-(337 \mathrm{MeV})^{-3}$. We shall take the strange sea quark to be degenerate with its valence counterpart, $m_{r}=m_{s}$, and fix the value at the physical strange quark mass. This leaves three parameters that can be varied, the valence pion mass, $m_{\pi \mathrm{val}}=m_{u u}$, the sea pion mass, $m_{\pi \text { sea }}=m_{j j}$, and the renormalization scale, $\mu$. While the $\mu$ dependence in the above expressions is exactly canceled by $\mu$-dependent counterterms at this order, we have not included these counterterms, nor do we have a reliable way to estimate them. Hence we vary $\mu$ to effectively obtain a range for the unknown counterterm contributions.

In Fig. 2, we assess the nonanalytic chiral corrections to the hyperfine splitting of nonstrange $\Xi^{*}$ and $\Xi$ baryons in PQ $\chi$ PT. To see the effect of partial quenching on this observable, we plot the difference between the chiral nonanalytic contribution to the hyperfine splitting in $\mathrm{PQ} \chi \mathrm{PT}$ and the corresponding nonanalytic correction in $\chi \mathrm{PT}$ :

$$
\delta \chi_{\mathrm{HF}}=\left(M_{\Xi^{*}}^{(3 / 2)}-M_{\Xi}^{(3 / 2)}\right)_{\mathrm{PQ} \chi \mathrm{PT}}-\left(M_{\Xi^{*}}^{(3 / 2)}-M_{\Xi}^{(3 / 2)}\right)_{\chi} \mathrm{PT},
$$

as a function of $m_{\pi \mathrm{sea}}$ for a few different values of $m_{\pi \mathrm{val}}$. For each value of $m_{\pi \mathrm{val}}$, we let $m_{\pi \mathrm{sea}}$ range from $m_{\pi \mathrm{val}}$ up to the mass of eta-strange, $m_{\eta_{s}}=m_{s s}=m_{r r} \approx 700 \mathrm{MeV}$. Thus the smallest value of $m_{\pi \text { sea }}$ corresponds to an unquenched theory at $m_{\pi}=m_{\pi \mathrm{val}}$, while the largest value of $m_{\pi \text { sea }}$ corresponds to a completely degenerate sea at the strange quark mass. The bands correspond to varying $\mu$ from $500 \mathrm{MeV}$ to $1500 \mathrm{MeV}$, which is chosen to be the same for both $\chi \mathrm{PT}$ and $\mathrm{PQ} \chi \mathrm{PT}$. For the $\chi \mathrm{PT}$ hyperfine splitting, we use the physical Goldstone boson masses, so by construction $\delta \chi_{\mathrm{HF}}=0$ when $m_{\pi \mathrm{sea}}=m_{\pi \mathrm{val}}=m_{\pi}$. As demonstrated by Fig. 2, chiral nonanalytic corrections to the hyperfine splitting are sizable, and increase with increasing $m_{\pi \text { sea }}$. Additionally corrections are enhanced as $m_{\pi \mathrm{val}}$ increases. While we do not trust the chiral expansion at large pion masses, we have kept these in the plot to show that chiral corrections can become large and tend to push the hyperfine splitting down by tens of MeV.

We can use our results to check the chiral corrections to the mass splitting relation predicted by heavy quarkdiquark symmetry $[1,8,9]$

$$
M_{\Xi^{*}}-M_{\Xi}=\frac{3}{4}\left(M_{P^{*}}-M_{P}\right) .
$$



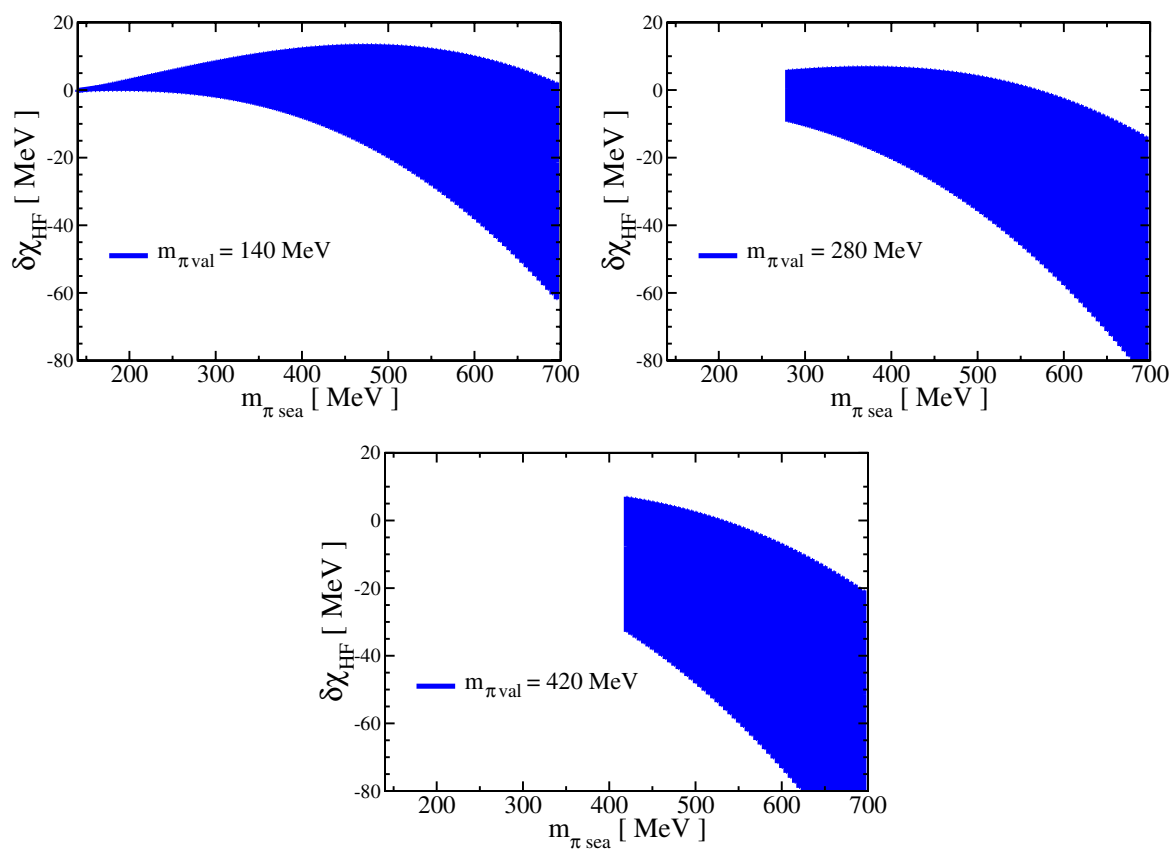

FIG. 2 (color online). $\quad \delta \chi_{\mathrm{HF}}$ as a function of $m_{\pi \mathrm{sea}}$ for different values of $m_{\pi \mathrm{val}}$. The width of the bands is the result of varying $\mu$ between $500 \mathrm{MeV}$ and $1500 \mathrm{MeV}$.

In Fig. 3, we plot the difference between the nonanalytic chiral corrections to this relation in $\mathrm{PQ} \chi \mathrm{PT}$ and $\chi \mathrm{PT}$, defined by

$$
\begin{aligned}
\delta \chi_{\mathrm{QDQ}}= & {\left[M_{\Xi^{*}}^{(3 / 2)}-M_{\Xi}^{(3 / 2)}-\frac{3}{4}\left(M_{P^{*}}^{(3 / 2)}-M_{P}^{(3 / 2)}\right)\right]_{\mathrm{PQ} \chi \mathrm{PT}} } \\
& -\left[M_{\Xi^{*}}^{(3 / 2)}-M_{\Xi}^{(3 / 2)}-\frac{3}{4}\left(M_{P^{*}}^{(3 / 2)}-M_{P}^{(3 / 2)}\right)\right]_{\chi^{\mathrm{PT}}},
\end{aligned}
$$

in the nonstrange sector. Here $M_{P^{*}}^{(3 / 2)}$ and $M_{P}^{(3 / 2)}$ are the chiral contributions to the masses of the vector and pseudoscalar heavy mesons, respectively [32]. As in Fig. 2, we

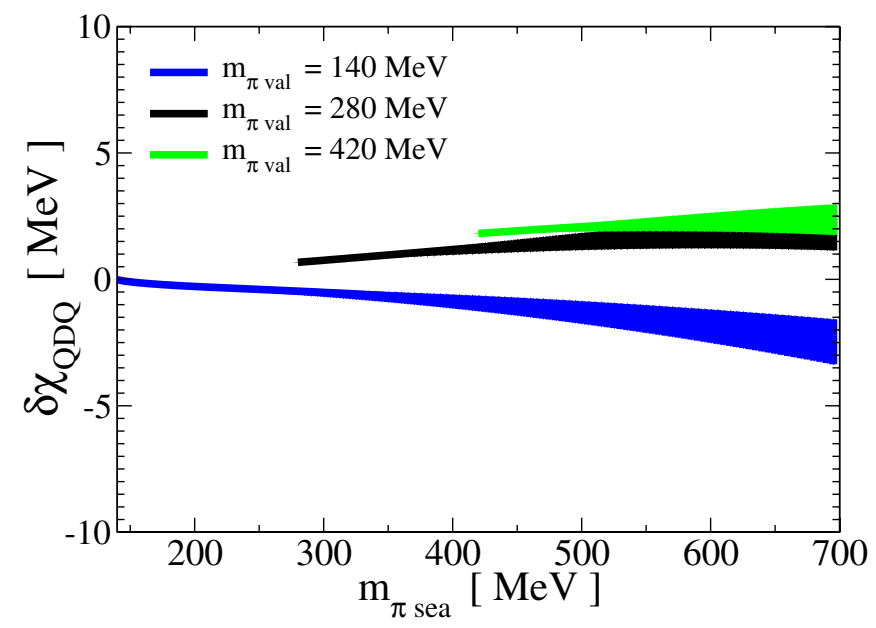

FIG. 3 (color online). $\quad \delta \chi_{\mathrm{QDQ}}$ as a function of $m_{\pi \mathrm{sea}}$ for different values of $m_{\pi \mathrm{val}}$. The width of the bands is the result of varying $\mu$ between $500 \mathrm{MeV}$ and $1500 \mathrm{MeV}$. vary $\mu$ from $500 \mathrm{MeV}$ to $1500 \mathrm{MeV}$ for three different values of $m_{\pi \mathrm{val}}$. Figure 3 shows that the chiral corrections to the heavy quark-diquark symmetry prediction are remarkably small compared to chiral corrections to the hyperfine splittings and much less sensitive to $\mu$. It was demonstrated in Ref. [13] that chiral corrections to the quark-diquark symmetry prediction for the hyperfine splittings are small and insensitive to $\mu$. Interestingly, this conclusion is not changed as the pion mass is increased, nor is it altered by partial quenching. While the hyperfine splittings of heavy mesons and doubly heavy baryons receive considerable chiral corrections for larger pion masses, the quark-diquark symmetry relation fortuitously does not. Lattice calculations of this quantity will not suffer large uncertainty due to chiral extrapolation.

\section{B. Q $\chi \mathbf{P T}$}

In the quenched theory we can similarly calculate the masses of doubly heavy baryons to one-loop order. The tree-level contribution from $S U(3)$ breaking counterterms is

$$
M_{\Xi_{a}^{*}}^{(1)}=M_{\Xi_{a}}^{(1)}=-\sigma^{Q}\left(m_{q}\right)_{a} .
$$

The tree-level baryon mass splittings are

$$
\begin{gathered}
\Delta_{b a}=M_{\Xi_{b}}-M_{\Xi_{a}}=M_{\Xi_{b}^{*}}-M_{\Xi_{a}^{*}} \\
=\sigma^{Q}\left[\left(m_{q}\right)_{a}-\left(m_{q}\right)_{b}\right], \\
\Delta_{b a}^{*}=M_{\Xi_{b}^{*}}-M_{\Xi_{a}}=\frac{3}{4} \Delta_{H}^{Q}+\sigma^{Q}\left[\left(m_{q}\right)_{a}-\left(m_{q}\right)_{b}\right] .
\end{gathered}
$$



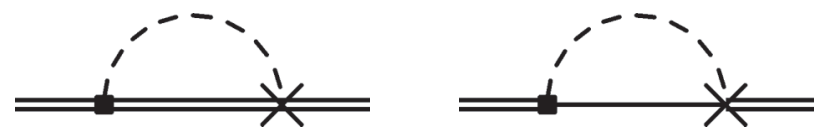

FIG. 4. Additional loop graphs which contribute to the masses of the doubly heavy baryons in $\mathrm{Q} \chi \mathrm{PT}$. The single and double lines correspond to $\Xi$ baryons or $\Xi^{*}$ baryons, respectively, while the dashed lines correspond to mesons. The filled squares denote the nonsinglet axial coupling, and the cross denotes the axial hairpin interaction given in Eq. (15).

The differences in low-energy constants notwithstanding, the one-loop mass contributions in $\mathrm{Q} \chi \mathrm{PT}$ are considerably altered from those in PQ $\chi \mathrm{PT}$ and $\chi \mathrm{PT}$. The sunset diagrams in Fig. 1 again contribute, however, it is clear that only hairpin contributions survive quenching. There are additional one-loop diagrams present in the quenched theory that have no counterparts in $\mathrm{PQ} \chi \mathrm{PT}$ or $\chi \mathrm{PT}$. These diagrams are depicted in Fig. 4 and involve the axial hairpin interaction in Eq. (15). Combining the hairpin diagrams and axial hairpin diagrams, we arrive at the oneloop quenched mass contributions

$$
\begin{aligned}
M_{\Xi_{a}^{*}}^{(3 / 2)}= & \frac{2 g^{Q} g^{\prime Q}}{(4 \pi f)^{2}}\left[\frac{5}{9} K\left(0, m_{a a}, \mu\right)+\frac{4}{9} K\left(-\Delta_{a a}^{*}, m_{a a}, \mu\right)\right] \\
& +\frac{\left(g^{Q}\right)^{2} \mu_{0}^{2}}{3(4 \pi f)^{2}} \frac{\partial}{\partial m_{a a}^{2}}\left[\frac{5}{9} K\left(0, m_{a a}, \mu\right)\right. \\
& \left.+\frac{4}{9} K\left(-\Delta_{a a}^{*}, m_{a a}, \mu\right)\right]
\end{aligned}
$$

for the spin-3/2 doubly heavy baryons, and

$$
\begin{aligned}
M_{\Xi_{a}}^{(3 / 2)}= & \frac{2 g^{Q} g^{\prime Q}}{(4 \pi f)^{2}}\left[\frac{1}{9} K\left(0, m_{a a}, \mu\right)+\frac{8}{9} K\left(\Delta_{a a}^{*}, m_{a a}, \mu\right)\right] \\
& +\frac{\left(g^{Q}\right)^{2} \mu_{0}^{2}}{3(4 \pi f)^{2}} \frac{\partial}{\partial m_{a a}^{2}}\left[\frac{1}{9} K\left(0, m_{a a}, \mu\right)\right. \\
& \left.+\frac{8}{9} K\left(\Delta_{a a}^{*}, m_{a a}, \mu\right)\right],
\end{aligned}
$$

for the spin- $1 / 2$ doubly heavy baryons.

With the quenched chiral nonanalytic corrections in hand, we can investigate their impact on the spectrum of

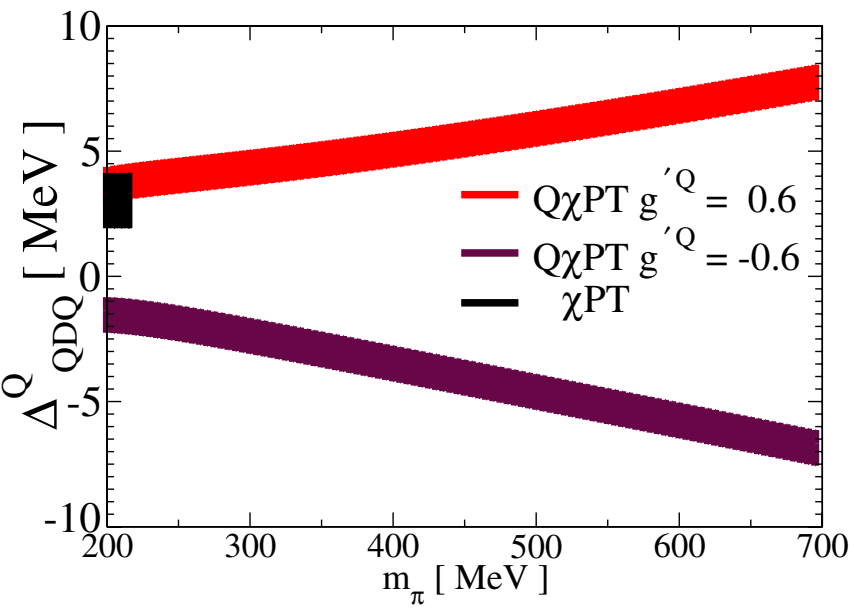

FIG. 6 (color online). The quenched chiral correction to the heavy quark-diquark symmetry breaking parameter, $\Delta_{\mathrm{QDQ}}^{Q}$, as a function of the quenched pion mass. The $\chi \mathrm{PT}$ band shows the corresponding range of chiral corrections to quark-diquark symmetry in QCD.

doubly heavy baryons as we did for the partially quenched theory. To determine these corrections, we must estimate the parameters in the quenched chiral Lagrangian. Little is known about these parameters. We adopt a central value of $\mu_{0}=700 \mathrm{MeV}$ from the analysis of Ref. [33]. The remaining parameters are taken to be the same values as in $\chi \mathrm{PT}$, though there is no justification for this. Finally we use two values, $g^{\prime Q}= \pm 0.6$, for the parameter that has no analogue in the unquenched theory. In Fig. 5, we plot the quenched chiral correction to the hyperfine splitting, $M_{\Xi^{*}}^{(3 / 2)}-M_{\Xi}^{(3 / 2)}$, as a function of the pion mass. The band plotted corresponds to a variation of $\mu$ from $500 \mathrm{MeV}$ to $1500 \mathrm{MeV}$. As in the partially quenched theory, we see that the mass splitting receives sizable chiral corrections that increase with the pion mass. The trend is the same as in the partially quenched theory provided $g^{Q}$ and $g^{\prime Q}$ have the same sign: quenched lattice calculations at larger pion masses than that in nature will underestimate the hyperfine splitting. When $g^{Q}$ and $g^{\prime Q}$ have opposite signs, it is possible for the splitting to be overestimated. Lastly we
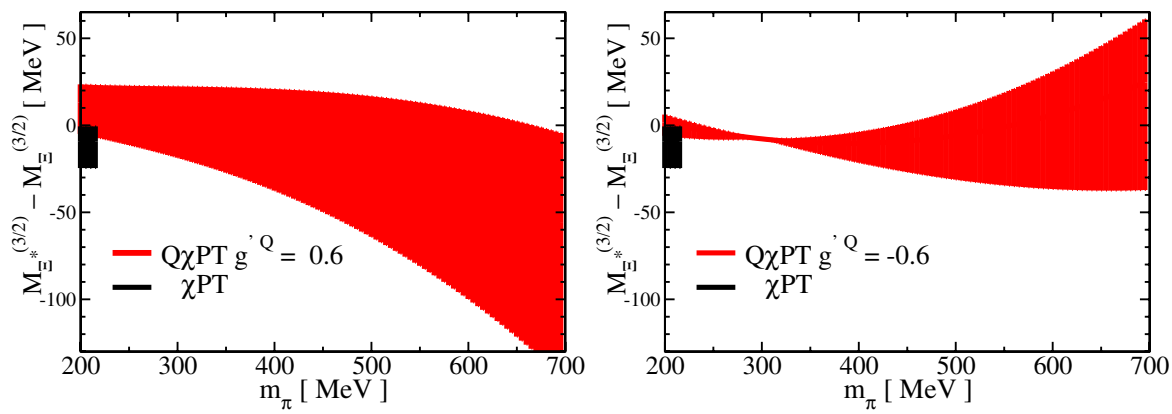

FIG. 5 (color online). The quenched chiral correction to the hyperfine splitting, $M_{\Xi^{*}}^{(3 / 2)}-M_{\Xi}^{(3 / 2)}$, as a function of the quenched pion mass. The $\chi \mathrm{PT}$ band shows the corresponding range of chiral corrections to the hyperfine splitting in QCD. 
can test how well heavy quark-diquark symmetry holds up in the presence of quenched chiral corrections. In Fig. 6, we plot the quenched chiral correction to the quark-diquark symmetry relation for the hyperfine splittings,

$$
\Delta_{\mathrm{QDQ}}^{Q}=M_{\Xi^{*}}^{(3 / 2)}-M_{\Xi}^{(3 / 2)}-\frac{3}{4}\left(M_{P^{*}}^{(3 / 2)}-M_{P}^{(3 / 2)}\right),
$$

as a function of the pion mass, again varying $\mu$ from 500 to $1500 \mathrm{MeV}$. Surprisingly, the quenched chiral corrections do not sizably alter the quark-diquark symmetry prediction. The corrections are only a few MeV. The $\mu$ dependence of the result is minimal and in fact one can show analytically that the $\mu$ dependence vanishes for this observable if $g^{\prime Q}=0$.

\section{ELECTROMAGNETIC TRANSITIONS IN PQ $\chi$ PT}

As a final application we determine the electromagnetic transition moments of the doubly heavy baryons in $\mathrm{PQ} \chi \mathrm{PT}$ and $\chi \mathrm{PT}$. To include electromagnetism into the theory, we merely gauge a background $U(1)$ field. In doing so, recall that the light-quark electric charge matrix $\mathcal{Q}$ is not uniquely defined in PQQCD [37]. The only constraint one must impose is that the charge matrix $\mathcal{Q}$ has vanishing supertrace. Following Refs. [38,39], we use

$$
\mathcal{Q}=\operatorname{diag}\left(q_{u}, q_{d}, q_{s}, q_{j}, q_{l}, q_{r}, q_{u}, q_{d}, q_{s}\right),
$$

along with the condition $q_{j}+q_{l}+q_{r}=0$. QCD is recovered in the limit of degenerate valence and sea quarks only for the particular choice: $q_{u}=q_{j}=\frac{2}{3}$, and $q_{d}=q_{s}=$ $q_{l}=q_{r}=-\frac{1}{3}$. Letting the charges be arbitrary, however, enables us to track the flow of charge in loop diagrams.

The relevant electromagnetic couplings in the chiral Lagrangian with quark-diquark symmetry are contained in the terms [13]

$$
\begin{aligned}
\mathcal{L}= & \frac{\beta e}{2}\left(\mathcal{H}^{\dagger} \mathcal{H} \mathcal{Q} \boldsymbol{\sigma} \cdot \boldsymbol{B}\right)+\frac{Q^{\prime} e}{2 m_{Q}}\left(\mathcal{H}^{\dagger} \tilde{\mathbf{\Sigma}} \cdot \boldsymbol{B} \mathcal{H}\right) \\
& +\frac{\beta_{E 2} e}{2 m_{Q} \Lambda_{\chi}}\left(\mathcal{H}^{\dagger} \tilde{\mathbf{\Sigma}}^{i} \mathcal{H} \mathcal{Q} \boldsymbol{\sigma}^{j}\right) \nabla_{\{i} \boldsymbol{E}_{j\}},
\end{aligned}
$$

where $Q^{\prime}$ is the charge of the heavy quark and the $5 \times 5$ matrices $\tilde{\boldsymbol{\Sigma}}$ are

$$
\tilde{\mathbf{\Sigma}}_{\mu \nu}=\left(\begin{array}{cc}
\boldsymbol{\sigma}_{\alpha \beta} & 0 \\
0 & -2 \mathcal{T}_{j k}
\end{array}\right) .
$$

The first term in Eq. (37) gives the leading contribution to the decay rate in the $1 / m_{Q}$ expansion. The contribution of this term at tree level is similar to the light quark magnetic moment contribution to the transition in the nonrelativistic quark model, i.e. the quark model reproduces the tree-level result of $\mathrm{HH} \chi \mathrm{PT}$ upon the identification $1 / m_{q} \rightarrow \beta$. The second term in Eq. (37) is the contribution from the magnetic moment of the heavy quark which is $O\left(1 / m_{Q}\right)$. The third term in Eq. (37) is also $O\left(1 / m_{Q}\right)$ suppressed. The notation $\{i j\}$ denotes symmetrization and tracelessness in the indices $i j$. In the heavy meson sector, this term contributes to the electric quadrupole moment of the $\boldsymbol{P}^{*}$ but not to the $\boldsymbol{P}^{*} \rightarrow P \gamma$ transition. It does give a contribution to the decay $\Xi^{*} \rightarrow \Xi \gamma$, which however is suppressed by $1 / m_{Q}^{2} \Lambda_{\chi}^{2}$ rather than $1 / m_{Q} \Lambda_{\chi}$ because it does not interfere with the leading-order contribution. Explicitly the formula for the decay width is given by

$$
\begin{aligned}
\Gamma\left(\Xi_{a}^{*} \rightarrow \Xi_{a} \gamma\right)= & \frac{4 \alpha}{9}\left[\left(\beta \mathcal{Q}_{a}-\frac{Q^{\prime}}{2 m_{Q}}+\delta \beta_{a}\right)^{2}\right. \\
& \left.+\frac{3}{4} E_{\gamma}^{2}\left(\frac{\beta_{E 2} \mathcal{Q}_{a}}{m_{Q} \Lambda_{\chi}}+\delta \beta_{E 2 a}\right)^{2}\right] \frac{M_{\Xi}}{M_{\Xi^{*}}} E_{\gamma}^{3},
\end{aligned}
$$

where $\delta \beta_{a}$ and $\delta \beta_{E 2 a}$ represent chiral corrections to the tree-level magnetic dipole and electric quadrupole transition moments, respectively. The loop diagrams shown in Fig. 7 give the leading nonanalytic chiral corrections.

The Lagrangian in Eq. (37) is easily extended to $\mathrm{PQ} \chi \mathrm{PT}$. Using the definition of the superfield $\mathcal{H}$ in Eq. (11), we can expand the Lagrangian in Eq. (37) to find the doubly heavy baryon part and thereby determine the contribution to the transition moments at tree level. As this contribution only involves valence quarks, the result is the same as in $\chi \mathrm{PT}$ [13]. The coefficients $\beta$ and $\beta_{E 2}$ have the same value as in $\chi \mathrm{PT}$ which can be demonstrated by matching to $\mathrm{PQ} \chi \mathrm{PT}$ in the sea sector. In the heavy quark limit, the operator proportional to $\beta_{E 2}$ can be neglected. When $O\left(1 / m_{Q}\right)$ hyperfine splittings are included in the loop diagrams, there are nonanalytic corrections to both $\beta$ and $\beta_{E 2}$. At one-loop order in PQ $\chi \mathrm{PT}$, we must calculate the diagrams shown in Fig. 7. Explicitly we find

$$
\begin{aligned}
\delta \beta_{a}= & -\frac{g^{2}}{24 \pi^{2} f^{2}} \sum_{b=j, l, r}\left(Q_{b}-\mathcal{Q}_{a}\right) \\
& \times \int_{0}^{1} d x\left\{\Delta_{1}(x) \log \frac{m_{a b}^{2}}{\mu^{2}}-m_{a b} R\left(\frac{\Delta_{1}(x)}{m_{a b}}\right)\right. \\
& \left.+5\left[\Delta_{2}(x) \log \frac{m_{a b}^{2}}{\mu^{2}}-m_{a b} R\left(\frac{\Delta_{2}(x)}{m_{a b}}\right)\right]\right\},
\end{aligned}
$$
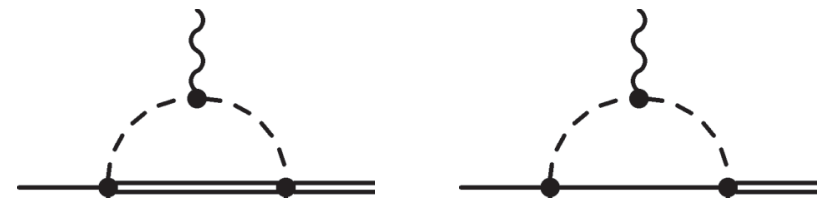

FIG. 7. One-loop graphs contributing to the doubly heavy baryon electromagnetic transitions in $\mathrm{PQ} \chi \mathrm{PT}$. The single lines correspond to $\Xi$ baryons, double lines to $\Xi^{*}$ baryons. The dashed lines correspond to mesons, while the wiggly lines correspond to photons. The coupling of Goldstone mesons to baryons is the nonsinglet axial coupling given in Eq. (16). 


$$
\begin{aligned}
\delta \beta_{E 2 a}= & \frac{g^{2}}{24 \pi^{2} f^{2}} \sum_{b=j, l, r}\left(Q_{b}-Q_{a}\right) \\
& \times \int_{0}^{1} d x x(1-x)\left\{\frac{\Delta_{1}(x) m_{a b}}{\left[\Delta_{1}(x)\right]^{2}-m_{a b}^{2}} R\left(\frac{\Delta_{1}(x)}{m_{a b}}\right)\right. \\
& \left.-\frac{\Delta_{2}(x) m_{a b}}{\left[\Delta_{2}(x)\right]^{2}-m_{a b}^{2}} R\left(\frac{\Delta_{2}(x)}{m_{a b}}\right)\right\},
\end{aligned}
$$

In expressing the transition moments, we have made the following definitions

$$
\begin{aligned}
& \Delta_{1}(x)=-\Delta_{a b}^{*}+x \Delta_{a a}^{*} \\
& \Delta_{2}(x)=-\Delta_{a b}+x \Delta_{a a}^{*},
\end{aligned}
$$

and used the function $R(x)$ defined in Eq. (25). The $\chi \mathrm{PT}$ result can be deduced by replacing the sea charges and sea masses with the values of their valence counterparts.

Reference [13] considered the loop corrections in $\chi \mathrm{PT}$ in the approximation where $\Delta_{H}=0$ and $\sigma=0$. This corresponds to taking the heavy quark limit and keeping only $O\left(\sqrt{m_{q}}\right)$ nonanalytic chiral corrections. In this limit the result can be inferred from the calculation of Refs. [40,41] for heavy mesons:

$$
\delta \beta_{a}=\frac{g^{2}}{4 \pi f^{2}} \sum_{b}\left(\mathcal{Q}_{b}-\mathcal{Q}_{a}\right) m_{a b} \quad \delta \beta_{E 2 a}=0 .
$$

For $\chi \mathrm{PT}$ with physical parameters, using Eq. (40) instead of Eq. (44) has little effect on the transition magnetic moment, $\beta \mathcal{Q}_{a}-Q^{\prime} /\left(2 m_{Q}\right)+\delta \beta_{a}$. The transition magnetic moment changes by $-14 \%(-1 \%)$ for $\mu=$ $500 \mathrm{MeV}(1500 \mathrm{MeV})$ for $a=1$ and by $-1 \%(+3 \%)$ for $\mu=500 \mathrm{MeV}(1500 \mathrm{MeV})$ for $a=2$. We conclude that keeping only the $O\left(\sqrt{m_{q}}\right)$ nonanalytic chiral correction is an adequate approximation for estimating the electromagnetic decay width of the $\Xi^{*}$ in QCD. Away from the physical value of the quark mass, chiral corrections become increasingly important and hence formulas in Eqs. (40) and (41) should be considered for lattice extrapolations.

The $x$-integrals can be evaluated analytically as shown in the appendix. In actual lattice calculations of transition moments, the current insertion method is limited by the discrete lattice momenta available. The matrix elements contributing to the electromagnetic decay are zero for vanishing photon three-momentum $\boldsymbol{q}$. If we wish to extract these matrix elements from a lattice simulation, it is necessary to know the momentum transfer dependence of the corresponding form factors in order to perform a model independent zero-momentum extrapolation. Formulas for the multipole transition form factors can be recovered from our expressions above by merely using the replacement

$$
m_{a b} \rightarrow \sqrt{m_{a b}^{2}-x(1-x) q^{2}},
$$

where $q^{2}$ is the virtuality of the photon.
While we do not wish to encourage quenched calculations of the transition moments, we shall comment briefly on the oddities of the quenched chiral calculation at oneloop order. None of the diagrams in Fig. 7 survive quenching. There is, however, quark mass dependence at this order, but it arises from the hairpin interactions depicted in Fig. 8. These diagrams are enhanced over their PQ $\chi \mathrm{PT}$ and $\chi \mathrm{PT}$ counterparts due to the size of the singlet parameter $\mu_{0}^{2}$. This was first observed for baryon magnetic moments in Ref. [42], and applied to baryonic transition moments in Ref. [43]. Thus in the chiral limit, the $\mathrm{Q} \chi \mathrm{PT}$ transition moments have chiral singularities

$$
\begin{aligned}
& \beta_{M 1}^{Q} \sim \beta_{M 1}^{(0) Q}-\frac{Q^{\prime}}{m_{Q}}+\alpha_{M 1} \mu_{0}^{2} \log m_{q}+\ldots \\
& \beta_{E 2}^{Q} \sim \beta_{E 2}^{(0) Q}+\alpha_{E 2} \mu_{0}^{2} \log m_{q}+\ldots,
\end{aligned}
$$

which should be contrasted with the chiral limit behavior in both PQ $\chi$ PT and $\chi$ PT

$$
\begin{aligned}
& \beta_{M 1} \sim \beta_{M 1}^{(0)}-\frac{Q^{\prime}}{m_{Q}}+\beta_{M 1}^{(1 / 2)} \sqrt{m_{q}}+\ldots \\
& \beta_{E 2} \sim \beta_{E 2}^{(0)}+\beta_{E 2}^{(1)} m_{q} \log m_{q}+\ldots,
\end{aligned}
$$

which are nonsingular.

The inclusion of dynamical quarks thus makes a dramatic difference in the chiral behavior of these observables. There are two types of dynamical contributions: those from the gauge configurations and those from the current operator self-contraction. The latter contributions are notoriously difficult to determine from the lattice. The effective theory can remedy this situation for the electromagnetic current in three-flavor simulations. Because we require $\operatorname{str} Q=0$, the choice $q_{j}=q_{l}=q_{r}=0$ is not ex-
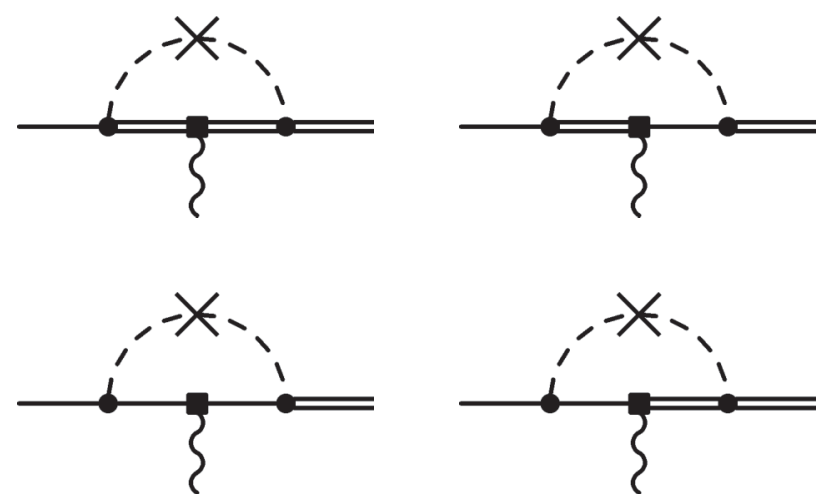

FIG. 8. Additional loop graphs for the doubly heavy baryon electromagnetic transitions in $\mathrm{Q} \chi \mathrm{PT}$. The single lines correspond to $\Xi$ baryons, double lines to $\Xi^{*}$ baryons. The dashed lines correspond to mesons, while the wiggly lines correspond to photons. The filled circles denote the nonsinglet axial coupling given in Eq. (15), the photon coupling is proportional to the magnetic couplings in Eq. (37), and crosses denote the quenched hairpin interaction. 
cluded, i.e. one can ignore the self-contraction of the current. In this case, one is not determining the physical transition moments, but rather the physical parameters $\beta$ and $\beta_{E 2}$ in the effective theory. These in turn can be used to make physical predictions.

\section{SUMMARY}

In this paper, we have extended the chiral Lagrangian with heavy quark-diquark symmetry to quenched and partially quenched theories. This extension allows for the derivation of extrapolation formulas necessary to connect lattice QCD data to the chiral limit. These formulas are easily modified to include the effects of finite volume, see Ref. [44]. Furthermore light-quark discretization effects can be incorporated, but depend on the particular fermion discretization employed in the lattice calculation.

As an example, we determined the quark mass dependence of doubly heavy baryons in both quenched and partially quenched chiral perturbation theory. We demonstrated that the hyperfine splitting between $\Xi^{*}$ and $\Xi$ baryons is sensitive to chiral nonanalytic corrections both in the quenched and partially quenched theories. Thus careful chiral extrapolation is required to connect lattice data to the doubly heavy baryon spectrum in the chiral regime. Surprisingly, we found that the heavy quarkdiquark symmetry relation is rather insensitive to chiral corrections. Despite the possible enhancement by factors of $\mu_{0}^{2}$ multiplying quenched chiral logarithms, this conclusion is true in the quenched theory.

We also investigated the chiral corrections to the electromagnetic transition moments. The width of the $\Xi^{*}$ baryons should be dominated by the decay $\Xi^{*} \rightarrow \Xi \gamma$. The lattice can be used to predict the electromagnetic decay widths and our results should be helpful for chiral extrapolations in these calculations. Since the doubly heavy baryon hyperfine splitting is expected to be $\approx$ $100 \mathrm{MeV}$, lattice pion masses will not allow on-shell intermediate states as one nears the chiral regime. This is in contrast to the $\Delta \rightarrow N \gamma$ transitions where the pion mass must be $m_{\pi} \geq 300 \mathrm{MeV}$ to avoid the multiparticle continuum. Furthermore, lattice data can test heavy quarkdiquark symmetry relations between the $\Xi^{*} \rightarrow \Xi \gamma$ and $P^{*} \rightarrow P \gamma$ decays.

Investigation of heavy mesons and doubly heavy baryons on the lattice provides a way to explore the implications of heavy quark-diquark symmetry. By varying the light quark mass, one will be able to see how well the predictions hold up against chiral corrections. Additionally by varying the heavy quark mass, one will be able to see in what regime this symmetry of the strong interaction emerges.

\section{ACKNOWLEDGMENTS}

B.C.T. would like to thank the Institute for Nuclear Theory at the University of Washington for hospitality and partial support during the course of this project. This work was supported by the U.S. Department of Energy under Grants No. DE-FG02-05ER41368 (T.M. and B.C. T.), DE-FG02-05ER41376, and DE-AC0584ER40150 (T. M.).

\section{APPENDIX}

Here we give some formulas which are useful for evaluating the $x$-integrals in Eqs. (40) and (41). A useful observation is that

$$
\frac{d R(x)}{d x}=\frac{x}{x^{2}-1} R(x)-2 .
$$

Using this it is straightforward to verify the following antiderivatives:

$$
\begin{aligned}
\int d x R(x) & =\frac{1}{8} \frac{1}{x^{2}-1} R^{2}(x)+\frac{x}{2} R(x)+\frac{x^{2}}{2}, \\
\int d x \frac{1}{x^{2}-1} R(x) & =-\frac{1}{4} \frac{1}{x^{2}-1} R^{2}(x), \\
\int d x \frac{x}{x^{2}-1} R(x) & =R(x)+2 x, \\
\int d x \frac{x^{3}}{x^{2}-1} R(x) & =\frac{x^{2}+2}{3} R(x)+\frac{2 x^{3}+12 x}{9} .
\end{aligned}
$$

All integrals in Eqs. (40) and (41) can be evaluated using these identities. For example, for one of the integrals appearing in $\delta \beta_{a}$, we find

$$
\begin{aligned}
& \int_{0}^{1} d x\left[\Delta_{1}(x) \log \left(\frac{m_{a b}^{2}}{\mu^{2}}\right)-m_{a b} R\left(\frac{\Delta_{1}(x)}{m_{a b}}\right)\right] \\
& =\frac{m_{a b}^{2}}{\Delta_{a a}^{*}} \int_{-\left(\Delta_{a b}^{*} / m_{a b}\right)}^{\left(\Delta_{a b}^{*}-\Delta_{a b}^{*}\right) / m_{a b}} d u\left[u \log \left(\frac{m_{a b}^{2}}{\mu^{2}}\right)-R(u)\right] \\
& \equiv \frac{m_{a b}^{2}}{\Delta_{a a}^{*}}\left[G\left(\frac{\Delta_{a a}^{*}-\Delta_{a b}^{*}}{m_{a b}}, \frac{m_{a b}}{\mu}\right)-G\left(-\frac{\Delta_{a b}^{*}}{m_{a b}}, \frac{m_{a b}}{\mu}\right)\right],
\end{aligned}
$$

where

$$
\begin{gathered}
G(x, y)=x^{2} \log (y)-\frac{x^{2}}{2}-\frac{1}{8} \log ^{2}\left(\frac{x-\sqrt{x^{2}-1+i \epsilon}}{x+\sqrt{x^{2}-1+i \epsilon}}\right) \\
-\frac{x \sqrt{x^{2}-1}}{2} \log \left(\frac{x-\sqrt{x^{2}-1+i \epsilon}}{x+\sqrt{x^{2}-1+i \epsilon}}\right) .
\end{gathered}
$$


[1] M. J. Savage and M. B. Wise, Phys. Lett. B 248, 177 (1990).

[2] A. V. Manohar and M. B. Wise, Cambridge Monogr. Part. Phys., Nucl. Phys., Cosmol. 10, 1 (2000).

[3] R. Lewis, N. Mathur, and R. M. Woloshyn, Phys. Rev. D 64, 094509 (2001).

[4] D. Ebert, R. N. Faustov, V. O. Galkin, and A.P. Martynenko, Phys. Rev. D 66, 014008 (2002).

[5] G. T. Bodwin, E. Braaten, and G. P. Lepage, Phys. Rev. D 51, 1125 (1995).

[6] M. E. Luke, A. V. Manohar, and I. Z. Rothstein, Phys. Rev. D 61, 074025 (2000).

[7] N. Brambilla, A. Pineda, J. Soto, and A. Vairo, Nucl. Phys. B566, 275 (2000).

[8] N. Brambilla, A. Vairo, and T. Rosch, Phys. Rev. D 72, 034021 (2005).

[9] S. Fleming and T. Mehen, Phys. Rev. D 73, 034502 (2006).

[10] M. B. Wise, Phys. Rev. D 45, R2188 (1992).

[11] G. Burdman and J.F. Donoghue, Phys. Lett. B 280, 287 (1992).

[12] T.-M. Yan et al., Phys. Rev. D 46, 1148 (1992).

[13] J. Hu and T. Mehen, Phys. Rev. D 73, 054003 (2006).

[14] T.D. Cohen and P. M. Hohler, hep-ph/0606084.

[15] M. A. Moinester et al. (SELEX), Czech. J. Phys. 53, B201 (2003).

[16] M. Mattson et al. (SELEX), Phys. Rev. Lett. 89, 112001 (2002).

[17] A. Ocherashvili et al. (SELEX), Phys. Lett. B 628, 18 (2005).

[18] V. V. Kiselev and A. K. Likhoded, hep-ph/0208231.

[19] B. Aubert et al. $(B A B A R)$, Phys. Rev. D 74, 011103 (2006).

[20] R. Chistov et al. (BELLE), hep-ex/0606051.

[21] N. Mathur, R. Lewis, and R. M. Woloshyn, Phys. Rev. D
66, 014502 (2002).

[22] J. M. Flynn, F. Mescia, and A. S. B. Tariq (UKQCD), J. High Energy Phys. 07 (2003) 066.

[23] A. Morel, J. Phys. (France) 48, 1111 (1987).

[24] S. R. Sharpe, Phys. Rev. D 46, 3146 (1992).

[25] C. W. Bernard and M. F. L. Golterman, Phys. Rev. D 46, 853 (1992).

[26] C. W. Bernard and M. F. L. Golterman, Phys. Rev. D 49, 486 (1994).

[27] S. R. Sharpe, Phys. Rev. D 56, 7052 (1997).

[28] M.F. L. Golterman and K.-C. Leung, Phys. Rev. D 57, 5703 (1998).

[29] S. R. Sharpe and N. Shoresh, Phys. Rev. D 62, 094503 (2000).

[30] S. R. Sharpe and N. Shoresh, Phys. Rev. D 64, 114510 (2001).

[31] P. H. Damgaard and K. Splittorff, Phys. Rev. D 62, 054509 (2000).

[32] S. R. Sharpe and Y. Zhang, Phys. Rev. D 53, 5125 (1996).

[33] W. A. Bardeen, E. Eichten, and H. Thacker, Phys. Rev. D 69, 054502 (2004).

[34] S. R. Sharpe, Phys. Rev. D 69, 034504 (2004).

[35] B. C. Tiburzi, Phys. Rev. D 71, 034501 (2005).

[36] S. Fajfer and J. Kamenik, hep-ph/0606278.

[37] J.-W. Chen and M. J. Savage, Phys. Rev. D 65, 094001 (2002).

[38] B. C. Tiburzi, Phys. Rev. D 71, 054504 (2005).

[39] W. Detmold and C. J. D. Lin, Phys. Rev. D 71, 054510 (2005).

[40] J. F. Amundson et al., Phys. Lett. B 296, 415 (1992).

[41] M. J. Savage, Phys. Rev. D 65, 034014 (2002).

[42] M. J. Savage, Nucl. Phys. A700, 359 (2002).

[43] D. Arndt and B. C. Tiburzi, Phys. Rev. D 69, 014501 (2004).

[44] D. Arndt and C. J. D. Lin, Phys. Rev. D 70, 014503 (2004). 